\title{
Efecto de los claros de bosque en la composición de murciélagos insectívoros en la Reserva Biológica la Tirimbina, Sarapiquí, Costa Rica
}

\author{
Effect of forest gaps on insectivorous bats composition in \\ Reserva Biológica La Tirimbina, Sarapiquí, Costa Rica
}

\author{
Priscilla Alpízar ${ }^{1,2}$, Luis R. Víquez-R ${ }^{2,3}$, Fernanda Hong ${ }^{1}$, \\ Bernal Rodríguez-Herrera ${ }^{1,2,5}$, José F. González-Maya ${ }^{3,4}$
}

\begin{abstract}
Resumen
Por las estrechas relaciones que pueden establecer con los ambientes que ocupan, los murciélagos han sido sugeridos como bioindicadores ideales de la perturbación de los ecosistemas naturales. Analizamos la composición de especies de murciélagos insectívoros aéreos, por medio de llamadas, en ambientes contrastantes como claros y bosques tropicales, con la finalidad de analizar la influencia de estas perturbaciones naturales sobre la composición de los ensambles. Durante mayo del 2009 se evaluó la presencia de murciélagos insectívoros por medio de un sistema de grabación ultrasónico de división de frecuencia. Se encontraron diferencias significativas en la composición entre bosques y claros (Jaccard=0,308), con una alta variación en la composición dentro de los sitios de bosque y una muy alta similitud entre los sitios de claros. Se discute el efecto potencial de los claros en la composición y uso por murciélagos insectívoros y el efecto potencial sobre el método de muestreo.
\end{abstract}

Palabras clave: Anabat; Claros; Jaccard; Insectívoros; Costa Rica.

\begin{abstract}
Due to the close relationships that bats establish with their environments, they have been considered ideal bioindicators for natural ecosystems' disturbance. We analyzed the species composition of aerial insectivorous bats, using echolocation calls, in gaps and inside tropical rainforests, in order to analyze the influence of these natural disturbances. During May 2009, we evaluated the presence of bats using a frequency division ultrasonic recording device. We found significant differences in species composition between the inside of the forest and gaps (Jaccard $=0.308$ ), and a high variation throughout the forests sites, and high similarities between the forest gaps. We discuss the potential effect of forest gaps in the insectivorous bats' composition and habitat use, as well as the effect of the sampling method.
\end{abstract}

Keywords: Anabat; Gaps; Jaccard; Insectivores; Costa Rica.

\section{Introducción}

Los murciélagos son capaces de explotar casi cualquier ambiente de la tierra, principalmente gracias a su capacidad de volar y al desarrollo de un efectivo sistema de ecolocalización que les permite encontrar alimento y orientarse en la oscuridad (Kalko y Schnitzler 1998). Dentro del grupo de los insectívoros aéreos (correspondiente en el neotrópico a las familias Emballonuridae, Molossidae, Vespertilionidae y Moormopidae), la variación presente en las llamadas de ecolocalización tiene una

\footnotetext{
Escuela de Biología, Universidad de Costa Rica, 2060 San José, San José, Costa Rica. e-mail: prialp55@gmail.com_fernandahong@gmail.com bernal.rodriguez@ucr.ac.cr

2 Theria Asociación para la Investigación y la Conservación de Mamíferos de Costa Rica, San José, Costa Rica. e-mail: luisviquez@gmail.com

3 Instituto de Ecología, Universidad Nacional Autónoma de México, 04510, México DF, México.

4 The Sierra to Sea Institute y ProCAT Colombia/Internacional, Las Alturas, Coto Brus, Costa Rica. e-mail: jfgonzalezmaya@gmail.com

5 Reserva Biológica La Tirimbina, Sarapiquí, Heredia, Costa Rica. Fecha recepción: Noviembre 20, 2012 Fecha aprobación: Diciembre 18, 2012
}

(c) Rev. Biodivers. Neotrop. 2012; 2 (2): 138-42 
fuerte relación con el tamaño del individuo, el tipo de presa que utiliza y el ambiente en el que éste se encuentre forrajeando (Jones y Holderied 2007). Las propiedades específicas de estas señales de ecolocalización (intervalo entre pulsos, barrido de frecuencias, frecuencia fundamental, número de armónicos, entre otros) proveen información al murciélago en términos de su posición y características del ambiente circundante (Mora et al. 2004). Tradicionalmente se ha establecido que las llamadas de los murciélagos varían con respecto a tres grandes categorías de ambientes: los espacios abiertos, de borde, y espacios cerrados (narrowed space), cada uno con características acústicas que determinan presiones de selección diferenciales al tipo de murciélago que puede explotar estas áreas de acuerdo con las restricciones establecidas por la flexibilidad que tengan sus llamadas (Schaub y Schnitzler 2007). Por la dinámica natural de los bosques, la presencia, número y distribución de claros están en constante cambio, influyendo directamente sobre el establecimiento de diferentes arreglos de especies en un área determinada. Los murciélagos son un componente fundamental de la fauna de las áreas boscosas; por tanto es importante identificar cómo estos mamíferos explotan los distintos microambientes, para poder determinar cómo las futuras modificaciones del bosque puedan afectar la composición de sus ensambles naturales. El objetivo principal de este estudio es analizar de manera preliminar la variación en la composición de especies de murciélagos insectívoros que vuelan dentro de bosques con cobertura de dosel y en los claros de bosque.

Se realizó un muestreo entre el 15 y el 30 de mayo del 2009 en la Reserva Biológica Tirimbina (RBT), distrito de Sarapiquí, provincia de Heredia, Costa Rica. La RBT se encuentra localizada en las tierras bajas del Caribe al noreste del país $\left(10^{\circ} 26^{\prime}\right.$ $\mathrm{N}, 83^{\circ} 59^{\prime} \mathrm{W}$ ) y cuenta con 345 ha protegidas de bosque húmedo tropical (BHT) (Holdridge 1979; Figura 1). La precipitación media anual de la zona llega a $3900 \mathrm{~mm}$, siendo noviembre, diciembre y febrero

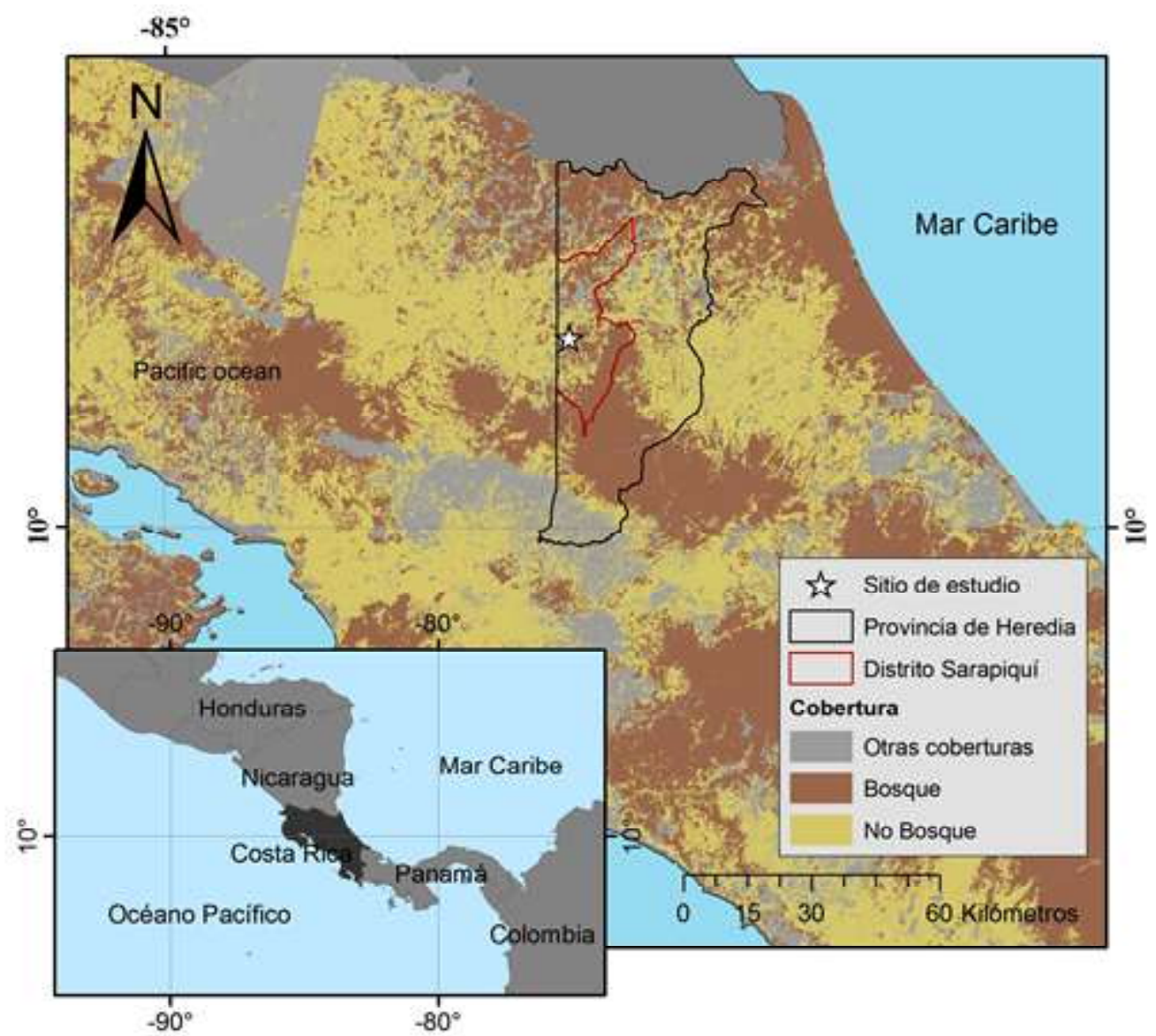

Figura 1. Localización del sitio de estudio, Reserva Biológica La Tirimbina, en el contexto nacional y regional. 
los meses más lluviosos para la zona (McDade et al. 1994). Para el muestreo se localizaron cinco claros dentro del bosque (se definió como claro a un espacio abierto natural dentro del bosque que tuviera más de $6 \mathrm{~m}$ de diámetro a nivel de dosel) a una distancia entre estos no menor de $60 \mathrm{~m}$. Para cada uno de los claros se seleccionó un punto de control dentro del bosque, situado al menos a $40 \mathrm{~m}$ desde el borde del claro. Tanto los claros como los puntos de control se encontraban sobre los senderos turísticos y de investigación de la RBT. Utilizando dos dispositivos de grabación ultrasónica por división de frecuencias ANABAT (Titley Electronics, Australia), se grabó de manera simultánea en bosque y claro por una noche desde las 17:30 hasta las 21:00 h. Los parámetros de grabación se fijaron en función del mejor desempeño de los equipos y la grabación (tasa de división=16, sensibilidad=6). La creación de los archivos se hizo de forma manual cuando se detectaba un murciélago en el sitio.

Para analizar los archivos se utilizó el software Analook (v.3.3q, Titley Electronics 2006) con la división del tiempo en F6. Para la identificación de las especies se utilizó literatura de referencia (Jung et al. 2007, O'Farrell et al. 1999, Rydell et al. 2002). Solo se incluyeron en los análisis a las familias de insectívoros aéreos Molossidae, Vespertilionidae, Emballonuridae y Mormoopidae. Se construyeron curvas de acumulación de especies utilizando como medida de abundancia relativa la ocurrencia de especies (Jung y Kalko 2011) y se seleccionó el modelo más ajustado (modelo de dependencia lineal, logarítmica o de Clench) por medio del criterio de información de Akaike (AIC) y el Cuadrado Medio del Error (CMError). Además, se calculó el índice de Jaccard para determinar qué tan similar era la composición de especies entre los claros y los puntos de bosque. Se realizó un dendrograma para evaluar la variabilidad dentro de bosques y claros con respecto al índice de Jaccard. Los análisis se realizaron en el paquete estadístico EstimateS 9 (Colwell 2013), y el software R (RTDC 2008).

En total se hicieron 869 grabaciones, 406 en los puntos de bosque y 463 en los claros. Se identificaron 13 especies de murciélagos, además de tres registros que sólo se lograron identificar a nivel de género (dos Myotis sp. y un Molossus sp.). Para los sitios de bosque los muestreos alcanzaron la asíntota de acumulación de especies (dependencia lineal, $\mathrm{p}<0,0001, \mathrm{a} / \mathrm{b}=2,67)$; sin embargo, para los claros esta no se alcanzó (dependencia lineal, $\mathrm{p}<0,0001$, a/ $b=6,9)$. La mayoría de las grabaciones ( $82 \%)$ fueron de las cuatro especies compartidas entre los dos sitios (Centronycteris centralis, Saccopteryx bilineata, Saccopteryx leptura y Cormura brevirostris). La composición de murciélagos insectívoros entre los claros y el bosque fue significativamente distinta (Jaccard=0.308), siendo mayor la riqueza de especies de murciélagos insectívoros en los claros que en el bosque (Tabla 1). Los claros mostraron a su vez una alta similitud, mientras los bosques fueron altamente heterogéneos entre sí (Figura 2).

Las cuatro especies de murciélagos que comparten los claros y el bosque son miembros de la

Tabla 1. Especies de murciélagos insectívoros aéreos registrados en la Reserva Biológica la Tirimbina, Sarapiquí, Costa Rica entre el 15 y 30 de mayo del 2009.

\begin{tabular}{clcc}
\hline Familia & \multicolumn{1}{c}{ Especie } & Claro & Bosque \\
\hline Emballonuridae & Centronycteris centralis & $\mathrm{X}$ & $\mathrm{X}$ \\
& Cormura brevirostris & $\mathrm{X}$ & $\mathrm{X}$ \\
& Diclidurus albus & $\mathrm{X}$ & \\
& Peropteryx kappleri & $\mathrm{X}$ & $\mathrm{X}$ \\
& Saccopteryx bilineata & $\mathrm{X}$ & $\mathrm{X}$ \\
& Saccopteryx leptura & $\mathrm{X}$ & $\mathrm{X}$ \\
Eptesicus brasiliensis & $\mathrm{X}$ & \\
& Myotis albescens & $\mathrm{X}$ & \\
& Myotis elegans & $\mathrm{X}$ & $\mathrm{X}$ \\
& Myotis nigricans & $\mathrm{X}$ & \\
Rhogeessa io & $\mathrm{X}$ & $\mathrm{X}$ \\
\hline Molossidae & Molossus molossus & & \\
\hline
\end{tabular}




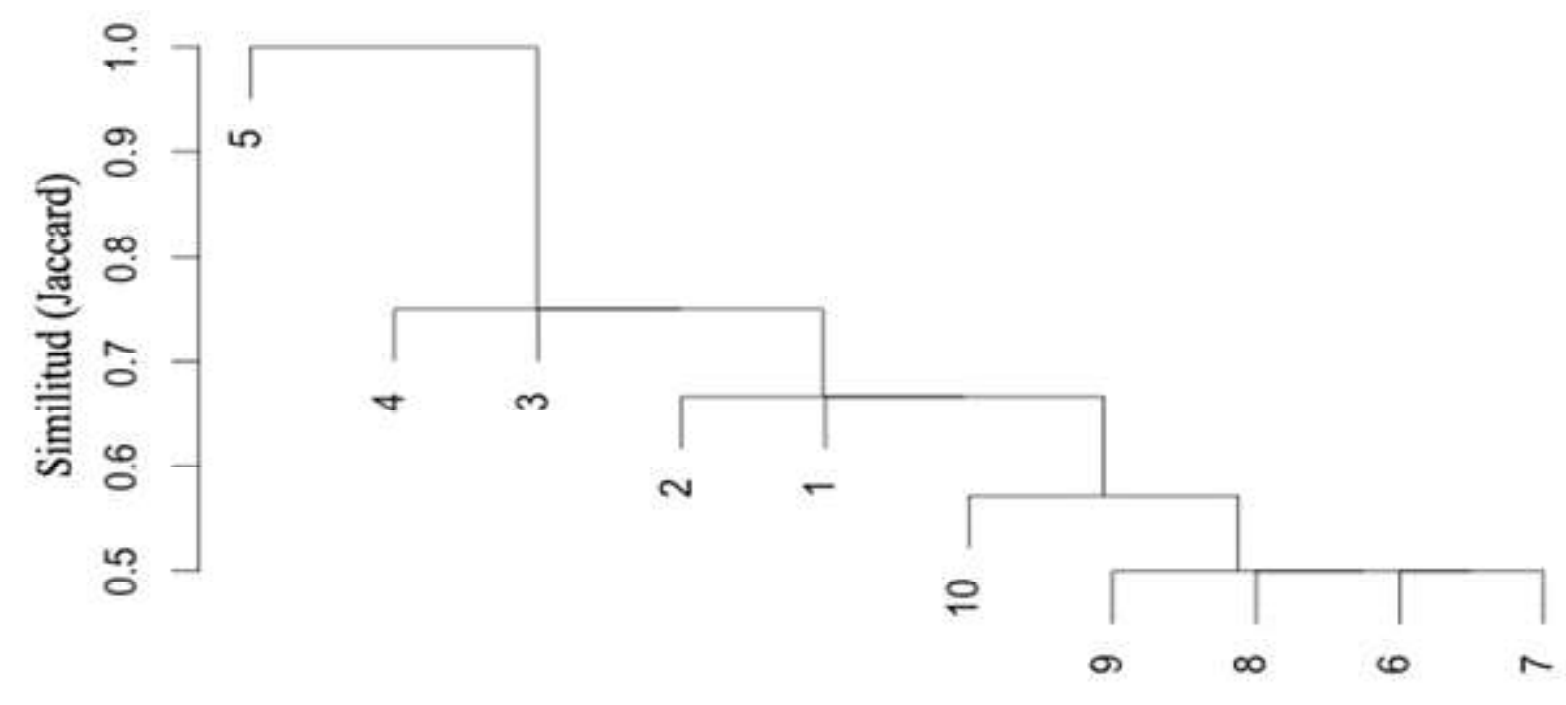

Sitios

Figura 2. Dendrograma jerárquico de similitudes de la composición de murciélagos insectívoros según el índice de Jaccard para cada sitio de muestreo en claros y bosque, Reserva Biológica La Tirimbina, Sarapiquí, Costa Rica. Sitios 1-5: Bosques y 6-10: Claros

familia Emballonuridae, que gracias al tipo de llamada que usan, pueden forrajear tanto dentro como fuera del bosque (Kalko 1995). Las otras nueve especies registradas en el trabajo sólo se encontraban en uno de los dos sitios, siendo algunas de estas detectadas en zonas coincidentes con sus historias naturales conocidas, por ejemplo Pteronotus parnelli estuvo asociada con zonas húmedas (Herd 1983) y Rhogeessa io, por su tamaño, se detectó solo en bosque, área que estaría funcionando como protección contra depredadores. La mayoría de las especies del género Myotis spp. se detectaron en zonas abiertas, porque sus llamadas (mayores a $40 \mathrm{kHz}$ y del tipo frecuencia modulada) son ideales para volar en espacios abiertos. Sin embargo, por la variabilidad en las llamadas en la familia Vespertilionidae, algunas especies como Myotis nigricans, pueden forrajear en zonas de bosque como se encontró en este estudio (Siemers et al. 2001).

En comparación con el interior del bosque, los claros ofrecen una serie de condiciones que facilitan el forrajeo por insectos por diferentes especies de murciélagos, porque la obstaculización del ambiente puede afectar tanto el área de vuelo como la maniobrabilidad de los murciélagos (Weller y Zabel 2002). En general, la vegetación siempre representa una causa de dispersión del sonido. Dentro de un mismo género, los murciélagos más grandes, con una mayor carga por su peso, presentan menores velocidades de vuelo y son más frecuentes en espacios cerrados (Schaub y Schnitzler 2007). Usualmente, especies de mayor tamaño producen llamadas con frecuencias más bajas, que pueden viajar mayores distancias sin sufrir tantas atenuaciones ambientales, y que no se ven tan afectadas por la vegetación (Jones y Rydell 2005, Siemers et al. 2001) y cuya resolución es mayor porque los ecos del fondo no interfieren con los generados por las presas (Schnitzler y Kalko 2001).

Aparte de las condiciones físicas y acústicas, los claros también pueden funcionar como rutas fijas de paso para los murciélagos. Por ejemplo, se ha demostrado que algunas especies de vespertiliónidos utilizan rutas fijas para salir de sus refugios, las cuales usualmente son límites entre espacios abiertos y bordes o cauces de ríos y que proporcionan puntos de referencia para su orientación (Schaub y Schnitzler 2007). En este sentido, los datos de este estudio se podrían explicar mejor si se considera que los claros de bosque pueden constituir para algunas especies puntos de referencia para la salida del bosque en la actividad de forrajeo en espacios abiertos y su regreso a los refugios.

En términos metodológicos, es importante men- 
cionar que existe además una posible influencia de la estructura de los ecosistemas sobre el rendimiento de los sistemas de grabación ultrasónicos por división de frecuencias, siendo más efectiva y eficiente la detección en hábitats abiertos que en el bosque, por lo que consideramos que el uso de metodologías complementarias (e.g. redes de niebla y grabación) deben ser utilizadas, aprovechando los alcances y deficiencias de uno y otro sistema para lograr inventarios completos de murciélagos.

Los resultados de este estudio, aunque preliminares, indican una alta variación en la composición de los murciélagos entre bosques y claros, así como un aparente uso diferencial selectivo de los mismos por parte de las especies de murciélagos insectívoros. Se sugiere considerar siempre el estudio de especies asociadas con espacios abiertos como claros y la implementación de la detección y análisis de frecuencias de eco como metodología complementaria para obtener listados de especies completos de la quiropterofauna en ecosistemas tropicales, así como para evaluar los ensambles de insectívoros en sistemas de ecotono y claros, porque estos pueden representar una alta heterogeneidad con respecto a sistemas continuos de bosque.

\section{Agradecimientos}

Los autores desean agradecer a todo el personal de la RBT y a Sergio Padilla y Beatriz Willink por su ayuda durante las noches de grabación. Marylin Bejarano y Andrés Arias-Alzate proporcionaron ayuda significativa en los análisis estadísticos y el manuscrito. Un agradecimiento especial a Eli Kalko, quien nos enseñó mucho de lo que sabemos de ecolocalización. Los autores Luis R. Víquez-R y José F. González-Maya agradecen a CONACyT México y UNAM por las becas de posgrado otorgadas (270173 y 255983 respectivamente) y a The Mikelberg Family Foundation por su apoyo. Gracias a Alex Mauricio Jiménez y Hugo Mantilla-Meluk por su asistencia en el proceso editorial.

\section{Literatura citada}

Colwell RK. 2013. EstimateS: Statistical estimation of species richness and shared species from samples. Version 9. User's Guide and application published at: http:// purl.oclc.org/estimates.

Herd RM. 1983. Pteronotus parnellii. Mammalian Species. 209: $1-5$.

Jones G, Holderied MW. 2007. Bat echolocation calls: adaptation and convergent evolution. Proc Royal Socy B: Biol Sci. 274: 905-12.

Jung K, Kalko EKV, von Helversen O. 2007. Echolocation calls in Central American emballonurid bats: signal design and call frequency alternation. J Zool. 272: 125-37.

Jung K, Kalko EKV. 2011. Adaptability and vulnerability of high flying Neotropical aerial insectivorous bats to urbanization. Div Distrib. 17: 262-74.

Holdridge L. 1979. Ecología: basada en zonas de vida. San José: Instituto Interamericano de Ciencias Agropecuarias (IICA).

Kalko EKV. 1995. Echolocation signal design, foraging habitats and guild structure in six neotropical sheath-tailed bats (Emballonuridae). Symp Zool Soc Lond. 67: 259-73.

Kalko EKV, Schnitzler HU. 1993. Plasticity in echolocation signals of European pipistrelle bats in search flight: implications for habitat use and prey detection. Behav Ecol Sociobiol. 33: 415-28.

Kalko EKV, Schnitzler HU. 1998. How echolocating bats approach and acquire food. En: Bat biology and conservation. Kunz TH, Racey PA (Eds.). Washington, DC: Smithsonian Institution Press. pp. 197-204.

McDade L, Bawa KS, Hespenheide HA, Hartshorn GS (Eds.) 1994. La selva: ecology and natural history of a Neotropical rain forest. Chicago: The University of Chicago Press. 486 pp.

Mora EC, Macías S, Vater M, Coro F, Kössl M. 2004. Specializations for aerial hawking in the echolocation system of Molossus molossus (Molossidae, Chiroptera). J Compar Physiol. A: Neuroethol, Sens, Neural, Behav Physiol. 190: 561-74.

RTDC-R team development core. 2008. R: A language and environment for statistical computing. Vienna: $\mathrm{R}$ Foundation for Statistical Computing.

Schaub A, Schnitzler H. 2007. Echolocation behavior of the bat Vespertilio murinus reveals the border between the habitat types «edge» and «open space». Behav Ecol Sociobiol. 61: 513-23.

Schnitzler HU, Kalko EKV. 2001. Echolocation by insect-eating bats. BioSci. 51: 557-69.

Siemers BM, Kalko EKV, Schnitzler HU. 2001. Echolocation behavior and signal plasticity in the Neotropical bat Myotis nigricans (Schinz, 1821) (Vespertilionidae): a convergent case with European species of Pipistrellus? Sociobiology 50: 417-28.

Weller TJ, Zabel CJ. 2002. Variation in bat detections due to detector orientation in a forest. Wildlife Soc Bull. 30: 92230 . 